\title{
El guion de ficción televisiva serial como producto
}

\section{Telesailetako fikziozko gidoiak produktu moduan}

\section{The TV series script as a product}

\section{Ángel Luis Lara Rodríguez ${ }^{1}$}

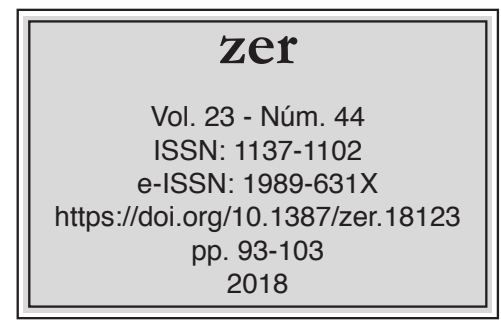

Recibido el 2 de octubre de 2017, aceptado el 2 de marzo de 2018.

\begin{abstract}
Resumen
El presente artículo se interroga acerca de la naturaleza del guion a partir de una concepción compleja del mismo que, aun entendiéndolo como eslabón en la cadena de materiales y tareas cuyo resultado es la serie de televisión como producto final, propone considerar los guiones como productos en sí mismos. Desde este prisma, la escritura de ficción televisiva aparece como un ámbito productivo cuyo resultado puede ser definido como producto susceptible de autonomía a partir de su condición de mercancía, independientemente de su traducción a la pantalla y de su conversión en contenido audiovisual de carácter televisivo.
\end{abstract}

Palabras clave: guion; series de televisión; economía política; narrativa audiovisual.

\section{Laburpena}

Artikulu honek gidoiaren izaerari buruz galdetzen du, haren ikuskera konplexutik abiatuta. Material eta zereginen kateko katebegi bat dela ulertuta ere, eta kate horren emaitza eta azken produktua telesaila dela ulertuta ere, gidoiak beraiek ere produktutzat hartzea proposatzen du. Ikuspegi horretatik, telebistako fikzioaren idazketa ekoizpen-mailan ageri da, eta merkantzia-izaera duen heinean, idazketa horren emaitza autonomia izan dezakeen produktu moduan defini daiteke, pantailara eraman eta telebistarako ikus-entzunezko eduki bihurtu edo ez bihurtu.

Gako-hitzak: gidoia; telesailak; ekonomia politikoa; ikus-entzunezko narratiba.

\begin{abstract}
Focusing on the analysis of the television series narrative in Spain, this article looks into the nature of scripts. Even though screenwriting is just a link in the chain of tasks and processes
\end{abstract}

1 laraa@oldwestbury.edu - State University of New York, College at Old Westbury 
resulting in a TV show as a final product, the text proposes to understand the TV series script as a product in itself. From this perspective, TV fiction screenwriting appears as an activity resulting in an autonomous product that works as a commodity in itself regardless of its translation to the screen.

Keywords: screenwriting; TV shows; political economy; audiovisual narrative. 


\section{Introducción}

Partiendo de una ubicación de la discusión acerca de la naturaleza del guion entre (1) un polo que entiende la escritura de historias televisivas y cinematográficas de ficción como una labor técnica cuyo único sentido viene determinado por la traducción de su contenido a la pantalla, y (2) un polo que le concede independencia y valor literario al guion por sí mismo, desarrollaremos una breve aproximación a la cuestión del sentido del guion de ficción televisiva como producto, que partirá de un acercamiento semántico al propio término "guion".

A partir de esta premisa, nos adentraremos en el ecosistema de la escritura de ficción televisiva serial en el Estado español proponiendo una conceptualización del guión como producto independiente. Parte importante de nuestro análisis será la referencia a la relación existente entre infravaloración de la cualidad de los guiones y condiciones laborales de los guionistas, marcadas por una invisibilización de los mismos. Igualmente, desplazaremos nuestra mirada hacia el campo de la economía política para descubrir, a partir del carácter mercantil de los guiones, la condición soberana e independiente que éstos presentan para la mayoría de los escritores empleados en los ciclos de fabricación de contenidos seriales de ficción televisiva.

\section{Función técnica y autonomía del guion: algunas cuestiones semánticas}

La consolidación del concepto de "guion", así como su incursión en los créditos de las películas, tuvo un momento importante en Hollywood a comienzos de los años treinta del pasado siglo, cuando los escritores de historias cinematográficas visibilizaron la importancia y la singularidad de su actividad. La creación explícita de un sindicato de guionistas en 1933, así como las primeras negociaciones entre escritores e industria para la consecución de un estatuto para la actividad de los primeros y su producto, jugaron un papel decisivo en este sentido.

Desde entonces, los ejercicios de conceptualización de la escritura audiovisual han dado lugar a una divergencia entre las posiciones que han primado la centralidad de la función técnica de los guiones en el conjunto del proceso de producción cinematográfica y las visiones que, más allá de la traducción audiovisual de los mismos a la pantalla, les han concedido autonomía y valor literario en sí mismos².

En los orígenes de estas conceptualizaciones, el guionista y dramaturgo Béla Balázs (1970: 248) construyó un viaje analítico por la evolución del guion partiendo de su inicial inexistencia, pasando por el uso del mismo como mero soporte técnico reducido a una lista de secuencias y tomas que orientaba la labor del director, para desembocar en lo que el autor húngaro denominó como "guion literario": un texto con valor estético y poético en sí mismo, cuya complejidad desborda toda reducción a un mero papel técnico. En el centro de esta propuesta analítica se colocó una evolución del lenguaje cinematográfico, así como de las condiciones técnicas de la expresión y la actividad fílmicas, que determinó una redefinición de la cualidad y del sentido de la escritura cinematográfica. Desde el punto de vista de Balázs, fueron la

\footnotetext{
2 Para una síntesis interesante acerca de esta divergencia se puede ver: Maras, 2009. Particularmente el capítulo "The screenplay as literature" (pp. 44-62).
} 
aparición del primer plano, ligado a una intensidad narrativa que propició un giro de las tramas y los personajes hacia la complejidad y la "psicologización" de las historias, así como la exigencia de diálogo a partir de la emergencia del cine sonoro, los elementos que propiciaron una suerte de "literaturización" de la escritura de guiones.

Sin embargo, la polémica disputa en torno a la cualidad del guion está lejos de resolverse a partir de la propuesta inicial de Balázs. De hecho, la complejidad asociada a la definición de la naturaleza de la escritura de guiones se encuentra en la propia semántica que funda la posibilidad de nombrar dicha actividad. Es en este orden semántico en el que podemos situar una complejidad ontológica a la hora de definir la cualidad y el sentido de la escritura de guiones.

Fue igualmente en Hollywood donde se localizó el desarrollo primigenio de los elementos claves en la evolución de la producción cinematográfica como una auténtica industria del cine, capaz de constituir el campo básico de referencia global en la producción audiovisual, tanto desde un punto de vista técnico y narrativo como estético. Dicha ubicación geográfica y cultural propició desde el inicio que el orden semántico de articulación de la conceptualización del cine se desarrollara netamente en inglés. De esta manera, sea cual sea el país o la cultura en la que ubiquemos nuestro análisis del medio audiovisual, siempre vamos a encontrar un léxico plagado de términos anglosajones y un universo estético que, de una manera u otra, ya sea para seguir su curso como para negarlo, toma constantemente la industria del cine estadounidense como referencia.

La sistematización de la escritura cinematográfica en Hollywood descansó en una conceptualización de la misma que le otorgó un carácter singular dentro del conjunto de las modalidades de escritura: no fue definida simplemente como writing, sino como scriptwriting. Es en el cruce entre el plano denotativo del término y lo que éste connota donde se puede identificar un elemento clave en torno a la cualidad del guion. Desde el punto de vista de la denotación, script significa tanto cualquier acto de escritura, como el objeto de la misma, mientras que en el explícito ámbito audiovisual la composición scriptwriting denota la acción concreta de escribir guiones, denominados en inglés como scripts ${ }^{3}$. Es, sin embargo, en el plano connotativo donde podemos localizar la implicación más relevante del término scriptwriting como definición de un tipo particular de escritura caracterizado por su naturaleza intermedia y por su cualidad meramente técnica: en el ámbito audiovisual el guion es concebido generalmente desde una perspectiva instrumental, no como un producto terminado, sino como una guía que prefigura y orienta el proceso de producción audiovisual y su resultado, ya sea éste una película, el episodio de una serie de televisión o un spot publicitario, por mencionar tan sólo algunos de los productos audiovisuales posibles.

Curiosamente, esta dimensión connotativa ha sido explorada con mayor intensidad en el campo de la telemática, hasta llegar a situarla en el plano de la denotación: en la programación informática los scripts son un conjunto de instrucciones, generalmente almacenadas en un archivo de texto, que deben ser interpretadas línea a línea en tiempo real para su ejecución. En este ámbito, scripting language se traduce como lenguaje de guion, un tipo de lenguaje de programación que implica una interpretación. Dicha definición muestra una simetría reseñable con la escritura audiovisual

\footnotetext{
El origen etimológico de script reside en el vocablo latino scriptum (algo escrito), que viene de scrībere (escribir).
} 
y aporta una descripción plenamente realista del papel del guion desde un punto de vista meramente técnico: debido a la cualidad de su contenido, el script, lejos de aparecer como un trabajo autónomo, subraya su carácter intermedio. No obstante, esta cualidad intermedia no se deriva únicamente de la dificultad manifiesta que presenta la consideración del guion como un producto terminado y autónomo, sino que tiene que ver también con la forma en la que la escritura de guiones se sitúa entre diferentes cualidades (industrial/artística), diversos órdenes mediáticos (texto escrito/audiovisual) y distintos procesos (escritura/producción audiovisual) (Maras, 2009: 48).

El anclaje semántico de la escritura de guiones, definida como una función técnica y de carácter intermedio en el seno de un proceso productivo cuyo resultado y sentido desbordan dicha actividad, se encuentra incluso más desarrollado en las categorías anglosajonas screenwriting (escritura de guiones) y screenplay (guion), en las que se vincula directa y explícitamente el sentido de la escritura con su traslación a la pantalla (screen). Desde esta perspectiva, toda autonomía resulta abolida y la traducción audiovisual del texto del guion se constituye en único sentido y producto, enunciándose una dependencia integral y absoluta de la actividad del guionista en relación a dicha traducción.

No obstante, es en la lengua castellana donde este universo de comprensión del guion encuentra tal vez un anclaje más sólido desde el punto de vista semántico. Así, el apelativo guion explicita y enfatiza su carácter de mera guía, proponiéndolo como un texto cuyo valor y sentido reside únicamente en exponer detalladamente el contenido y la forma de su puesta en escena ${ }^{4}$.

Sin embargo, esta centralidad de la escenificación en la conceptualización del sentido y el rol de la escritura audiovisual va más allá de la mera esfera semántica, conectándose con dos de los patrones convencionales de organización de la producción cinematográfica y televisiva. El primero de esos patrones está constituido por la separación entre concepción y ejecución: el guion es concebido como una especie de máquina abstracta que prescribe una práctica, como material que guía una ejecución ${ }^{5}$. El segundo de los patrones es un carácter lineal del proceso productivo que encadena tres fases fundamentales de actividad: preproducción, producción, posproducción. A partir de este esquema, las tareas de escritura se situarían en la fase de preproducción, mientras que la etapa posterior de producción sería concebida como ejecución del guion siguiendo una lógica que dibujaría un movimiento que va del concepto al trabajo finalizado (from script to screen - del guion a la pantalla-).

Lo paradójico de esta organización de la producción estructurada a partir de la distinción y segmentación entre concepción y ejecución es que funciona como base ambivalente para la argumentación en favor de posiciones encontradas. Por un lado, alimenta los puntos de vista que le niegan al guion todo carácter autónomo, puesto que en el esquema de escisión entre concepción y ejecución el guion únicamente funciona como texto-guía cuyo sentido viene determinado enteramente por su escenificación.

\footnotetext{
${ }^{4}$ La puesta en escena como horizonte de sentido del texto de un guion se pone explícitamente de manifiesto en los apelativos con los que se nombra la palabra "guion" en francés (scenario), italiano (sceneggiatura) y ruso (сценарий -stsenari-).

5 Deleuze y Guattari describen la máquina abstracta o diagramática como aquella que no funciona para representar, ni siquiera cualquier cosa real, sino para construir algo real en ciernes, un tipo diferente de realidad (1994: 519-522).
} 
Por otro lado, sin embargo, la mencionada escisión puede servir como terreno de justificación de aquellas posiciones que le conceden autonomía y soberanía al guion en base tanto a su carácter originario y previo, como a su cualidad prescriptiva y performativa en relación al proceso de producción y al producto final de dicho proceso ${ }^{6}$. Tanto en un caso como en otro, la separación entre concepción y ejecución aparece como eje que articula los discursos en torno a la producción cinematográfica y televisiva de contenidos de ficción, así como a la organización de la misma.

\section{En torno al carácter soberano del guion}

La cualidad performativa del guion en relación al proceso de producción de las ficciones audiovisuales, así como su carácter preliminar y motor en el proceso concreto de fabricación de las películas o las series de televisión, hace posible tanto una conceptualización de la escritura que subraye su centralidad e importancia, como una concepción del guion a partir de su carácter soberano. Por un lado, los guiones son objetos que presentan la forma de textos terminados en sí mismos. Por otro lado, el guion se dota de una suerte de halo trascendental: estipula y dicta a priori lo que va a pasar en el set de rodaje y, más allá, determina cada una de las fases de la producción y la postproducción.

Sin embargo, esta idea de la soberanía del guion resulta enormemente problemática en el caso de la producción de contenidos de ficción para televisión. La identificación de la concepción y la ejecución como dos esferas separadas de actividad posee escaso valor explicativo en el ámbito televisivo: el guion no es tanto un objeto terminado como un proceso continuo en el que el texto, así como las pautas de su escenificación prescritas por los escritores, se van modificando por la acción de los otros agentes que intervienen en las diferentes fases del proceso de fabricación de una serie de ficción televisiva?.

La elaboración de guiones televisivos no solamente posee una naturaleza altamente procesal, sino que muestra un carácter tan sumamente dinámico que nos obliga a poner el énfasis en los procesos y las actividades implicadas en la escritura de guiones, más que fijar la mirada en el manuscrito terminado. En este sentido, el guion aparece siempre como un work in progress, como el espacio de una reelaboración permanente cuyo alcance posee la cualidad de una verdadera conceptualización constante. Desde este punto de vista, la definición trascendental del guion como un objeto acabado choca con una realidad productiva que difiere por completo de esa concepción del producto de la escritura televisiva.

No obstante, tampoco conviene dejarse llevar en exceso por las pautas de comprensión de la escritura de guiones que le conceden únicamente un valor técnico, reduciendo su papel a la confección de una mera guía. Es importante remarcar que la escritura de guiones implica el desarrollo de una constante labor creativa e

\footnotetext{
${ }^{6}$ Sobre el concepto de "soberanía del guion" se puede ver: Bordwell, Staiger y Thompson, 1985; y Maras, 2009.

7 Como dato curioso al respecto se puede señalar que hay guionistas televisivos que prefieren no ver la emisión de los episodios seriales que escriben, dado que el texto elaborado por ellos suele sufrir tantas modificaciones a lo largo del proceso productivo que su traducción a la pantalla dista mucho de aquello que habían imaginado y escrito.
} 
imaginativa enfocada a la constitución de universos narrativos. Por más que dicha actividad se traduzca en última instancia en la elaboración de textos que desde un prisma técnico poseen un carácter intermedio, tanto el proceso como el producto del trabajo de los guionistas se configura a partir de elementos de naturaleza eminentemente narrativa y literaria: arcos dramáticos, personajes, tramas, giros, argumentos, cliffhangers $^{8}$, etc.

Conviene, por tanto, matizar las posiciones que, anclando sus análisis en una cuestionable separación entre concepción y ejecución, enfatizan la cualidad técnica del guion en detrimento de su dimensión narrativa. Al mismo tiempo, resulta conveniente poner en relación el menosprecio del valor literario de la escritura de ficción televisiva con las relaciones de poder que atraviesan dicho ecosistema narrativo. Desde este punto de vista, tanto la separación de concepción y ejecución, como la negación del valor artístico de la escritura de guiones televisivos, se relacionan con las pautas de invisibilización y menosprecio del trabajo de los escritores en el ámbito de la fabricación de las series de televisión. Dicha invisibilización no sólo implica lo que el guionista y director Philip Dunne (1980: 42) definió como "un problema de reconocimiento y de prestigio", sino que se constituye en dispositivo estratégico de peso en el gobierno patronal de los escritores de ficción televisiva serial en Estado español.

Pese a que los guionistas operan como una variable invisible para las audiencias, los medios de comunicación o las estrategias de marketing de las cadenas españolas, constituyen una figura clave que habita en el centro mismo del ciclo productivo de los relatos seriales de ficción para televisión. La configuración de los imaginarios asociados a las series televisivas en Estado español, así como la mercadotecnia que los produce y los estira para crear y fidelizar públicos, giran en torno a otros factores como la popularidad de los actores y las actrices protagonistas o la dimensión de la producción cuando se trata de un producto con un alto presupuesto. De esta manera, los guionistas habitan en el ámbito de las series televisivas una zona de sombra que recubre los procesos productivos de los que son parte relevante: como sucede con el conjunto de las mercancías que llenan las repisas de los supermercados o las plataformas de venta electrónica en Internet, las historias de ficción televisiva parecen tener una voluntad independiente a sus creadores, como si brotaran directamente de la pantalla cuando encendemos los televisores, hacemos zapping con nuestro mando a distancia, nos proveemos de algún contenido en alguna red de descarga digital o seguimos nuestra serie favorita en el teléfono móvil.

Esta invisibilidad de los guionistas empleados en los ciclos de fabricación de las series de televisión en Estado español, además de contribuir a provocar en los públicos la ilusión de un producto sin productores, se desvela como factor que incide en la configuración del ethos de los propios escritores, generando consecuencias materiales y anímicas que afectan al modo y a las condiciones en las que desarrollan su labor profesional, así como a sus estados emocionales y a las pautas de configuración de su identidad colectiva. Invisibilizar el guion y concebir la creación de historias y la escritura de guiones de ficción televisiva como una mera tarea técnica de carácter

\footnotetext{
8 Un cliffhanger (literalmente "colgando de un acantilado" o "al borde del precipicio") es un artificio narrativo mediante el cual la escena que normalmente, al final del capítulo de una obra de ficción que se espere que continúe en otra entrega, genera el suspense o la conmoción necesaria para hacer que el lector o auditor se interese en conocer el resultado o desarrollo de dicho efecto en la siguiente entrega.
} 
intermedio, sin cualidad artística ni valor en sí mismo, contribuye inevitablemente a una desvalorización de los escritores ${ }^{9}$.

\section{Guion televisivo y forma mercancía: producto y relaciones de producción}

A la hora de reflexionar acerca del carácter autónomo del guión de ficción televisiva, así como de su posible soberanía, dos preguntas inevitables emergen de inmediato: ¿por qué hablar del guión como un proceso en sí o como un producto acabado si no constituye más que una pieza incompleta de una obra mayor en la que se inserta? ¿No es la serie de televisión el verdadero producto? Dotar de sentido la propuesta de comprensión de la escritura de ficción televisiva como un ámbito productivo en sí, cuyo resultado puede ser definido en los términos de un producto susceptible de autonomía, requiere que abramos el razonamiento, desplazándolo hacia una de las características de los guiones televisivos actuales que, pese a su carácter fundamental, no resulta siempre evidente en los análisis: los guiones son mercancías. Es el prisma de la economía política de la escritura televisiva, así como el carácter mercantil de ésta, el que permite entender de manera más eficaz como el guion es susceptible de funcionar en tanto que producto independiente de su conversión en contenido audiovisual de carácter televisivo, es decir, de su traducción a la pantalla.

Esta apertura analítica, que desplaza la mirada más allá tanto del trabajo concreto de los guionistas como del contenido material de su resultado, encuentra un estímulo inicial en una conferencia impartida por Walter Benjamin el 27 de abril de 1934 en el Instituto para el Estudio del Fascismo que los emigrantes alemanes, expulsados por la persecución nacionalsocialista, habían fundado en París. Entre las muchas claves que ofrece esta conferencia, antecedente directo del famoso ensayo sobre la obra de arte, destaca para nosotros un pasaje en el que Benjamin realiza un aporte esencial desde el puto de vista metodológico:

Antes de la pregunta: ¿cuál es la actitud de una obra frente a las relaciones de producción de la época?, quisiera preguntar: ¿cuál es su posición dentro de ellas? Esta pregunta apunta directamente hacia la función que tiene la obra dentro de las relaciones de producción literarias de una época. Con otras palabras, apunta directamente hacia la técnica literaria de las obras (Benjamin, 2004: 25).

Siguiendo la deriva metodológica propuesta por Benjamin, es en la posición que ocupa el guion dentro de las relaciones de producción propias de la fabricación de series de ficción televisiva en donde encontramos una clave para rastrear el carácter soberano y autónomo de los guiones. Junto a la pregunta acerca del qué de la escritura de ficción televisiva, conviene pues centrar la atención en el cómo y el para $q u e ́$ de dicha actividad. Desde este prisma, el trabajo de los escritores aparece como una esfera mercantilizada y el producto-guion viene definido en los términos de

9 Para un análisis pormenorizado sobre este asunto se puede ver: Lara, 2018. 
una mercancía que resulta intercambiada en un mercado. Para entender este carácter directamente mercantil del producto de la escritura televisiva resulta útil aterrizar el análisis en el régimen múltiple y dislocado en el que se desenvuelve la relación entre los guionistas y las empresas de producción de contenidos para televisión.

Una de las características fundamentales del ecosistema profesional en el que se desenvuelve la escritura de historias seriales de ficción para televisión en Estado español es la generalización del trabajo por cuenta propia. Según datos aportados por un estudio cuantitativo sobre la condición de los guionistas españoles publicado por la Fundación Autor en 2012, el 62,3\% de los escritores audiovisuales españoles declaraba aquel año realizar su actividad laboral inscrito en el Régimen Especial de Trabajadores Autónomos (RETA), mientras que tan sólo el 29\% de los guionistas cotizaba a la Seguridad Social como asalariado en régimen general. Entre los guionistas empleados en los ciclos de fabricación de series de ficción televisiva, solamente un $17,1 \%$ declaraba recibir una remuneración sujeta formalmente a una relación salarial, mientras que el 64,6\% de ellos obtenía sus honorarios únicamente por obra realizada, sujetos a un régimen permanente de intermitencia e irregularidad en la prestación laboral (Fundación Autor, 2012) ${ }^{10}$. Pese a que no existen datos más actualizados al respecto, en base a mi experiencia en el sector, así como a las continuas conversaciones con escritores empleados en los ciclos de fabricación de contenidos televisivos, se puede concluir que la tendencia apuntada por la Fundación Autor se ha consolidado plenamente en nuestros días.

A partir de estas premisas, la regulación de la relación entre los escritores y la organización empresarial en los ciclos de producción de ficción televisiva serial presenta dos modalidades diferenciadas:

a) Una relación laboral clásica regulada mediante un contrato de trabajo entre el demandante y el dador de empleo. Esta modalidad se desarrolla a través de contratos fijos, prácticamente inexistentes y puramente anecdóticos en el conjunto de las contrataciones que se operan en el sector de la producción de ficción televisiva, y contratos por obra, ligados a la suerte y las necesidades de los ciclos de fabricación del producto en el que el guionista desempeñe su actividad.

b) Una relación mercantil de servicio en la que el guionista, sujeto al estatuto jurídico de trabajador autónomo, vende sus servicios como escritor de contenidos televisivos a una empresa productora. Esta modalidad, mayoritaria en el conjunto del sector audiovisual, ubica la escritura de ficción televisiva en un espacio absolutamente diferente al de las relaciones laborales, originando que los guionistas vean su figura profesional excluida de la tutela que tradicionalmente ofrecía el Derecho del Trabajo.

\footnotetext{
${ }^{10}$ El estudio de la Fundación Autor enfatiza la inestabilidad propia del desempeño del trabajo de guionista a partir de un dato: el $40 \%$ de los escritores desarrollaba su actividad, al menos en ocasiones, sin la cobertura de un contrato, lo que indica la precariedad laboral que caracteriza al sector. El estudio citado únicamente aborda la condición de los escritores audiovisuales españoles desde un prisma cuantitativo. Para un análisis complejo y en profundidad de los guionistas de ficción televisiva en Estado español se puede ver: Lara, 2013.
} 
El hecho de que la mayoría de los escritores estén sujetos a esta segunda modalidad de regulación, con una prestación laboral anclada en una relación explícita de servicio, les ubica fuera de la racionalidad propia de las relaciones salariales tout court, en las que el trabajador viene remunerado por el uso patronal de su fuerza de trabajo. A diferencia de este modelo tradicional, la mayoría de los guionistas empleados en los ciclos de fabricación de contenidos seriales para televisión reciben su remuneración a partir de la venta del producto de su trabajo, el guion, al empleador correspondiente. De este modo, lo que el régimen de trabajo por cuenta propia determina es la configuración de una relación mercantil entre el trabajador y la empresa cuyo vínculo se establece a partir de la compra de la mercancía guion por parte de la compañía productora. Dicha mercancía, por tanto, se realiza para el guionista no tanto a partir de su traducción audiovisual final en la pantalla, como de su venta a una empresa que opera en los términos de un cliente.

Desde este prisma, por tanto, el guión puede ser entendido como un verdadero producto cuya cualidad viene definida por el carácter dual que presenta toda mercancía: su utilidad lo convierte en valor de uso y este es, además, el soporte de un valor de cambio. Marx ofrece una definición básica en este sentido que se ajusta convenientemente al caso de la escritura de contenidos de ficción televisiva:

La mercancía es, en primer término, un objeto externo, una cosa apta para satisfacer necesidades humanas, de cualquier clase que ellas sean. El carácter de estas necesidades, el que broten por ejemplo del estómago o de la fantasía, no interesa lo más mínimo para estos efectos. Ni interesa tampoco, desde este punto de vista, cómo ese objeto satisface las necesidades humanas, si directamente, como medio de vida, es decir, como objeto de disfrute, o indirectamente, como medio de producción. (...) La utilidad de un objeto lo convierte en valor de uso. (...) Lo que constituye un valor de uso o un bien es, por tanto, la materialidad de la mercancía misma (...) El valor de uso sólo toma cuerpo en el uso o consumo de los objetos. (...) En el tipo de sociedad que nos proponemos estudiar, los valores de uso son, además, el soporte material del valor de cambio. A primera vista, el valor de cambio se presenta como la relación cuantitativa, la proporción en que se cambian valores de uso de una clase por valores de uso de otra (...) (Marx: 1981: 3).

El primer pasaje de esta mínima definición de la mercancía señala que el valor de uso, es decir, la utilidad del producto, se orienta en dos sentidos fundamentales: convierte la mercancía en (1) objeto de disfrute o en (2) medio de producción. En el primer ámbito, tal y como hemos visto en páginas anteriores, el carácter intermedio del guion hace que el objeto de disfrute no esté constituido por el guion en sí, sino por el producto terminado de ficción televisiva serial. Por decirlo de manera más sencilla, lo que los públicos televisivos disfrutan, por usar la terminología que emplea el propio Marx, es el episodio de la serie como puesta en escena y traducción audiovisual del contenido del guion, no el producto-guion en sí. De esta manera, la escritura de ficción televisiva produce un texto cuyo valor de uso reside en su 
condición de medio de producción del producto terminado de serie de televisión en el que se inserta. Desde este punto de vista, los guiones se presentan como medios de producción, es decir, como recursos generados con el fin de posibilitar la producción de un bien, en el caso concreto que nos ocupa, una serie de ficción televisiva.

Sin embargo, el medio televisivo añade un grado mayor de complejidad al razonamiento que acabamos de proponer, puesto que el producto fundamental que fabrica la industria televisiva es la propia audiencia de sus programas: los contenidos televisivos operan en realidad como medios de producción de las audiencias, verdaderas mercancías que son vendidas en el mercado publicitario y que funcionan como el elemento más relevante del negocio televisivo. Las cadenas de televisión fidelizan a los telespectadores a partir de los contenidos que emiten, agrupándolos en paquetes de audiencias que venden a las compañías publicitarias, quienes a su vez colocan sus anuncios en el bucle de la emisión televisiva para atrapar la atención de esas audiencias. Desde este punto de vista, el propósito de los contenidos seriales de ficción televisiva no es otro que confeccionar audiencias para ser vendidas. En este esquema, los guiones de televisión constituyen medios de producción para la fabricación de unos contenidos de ficción que, pese a contener un incuestionable valor de uso para los públicos televisivos, operan en realidad como medios de producción de las audiencias televisivas.

A tenor de lo expuesto, el valor de uso de los guiones de ficción para televisión hace que se conviertan en medios de producción, subrayando el carácter intermedio y técnico de los mismos. Sin embargo, como apunta Marx en su definición básica de la mercancía, todo valor de uso funciona como soporte de un valor de cambio, entendiendo éste como el valor que un producto tiene en el mercado y que se expresa en términos cuantitativos, medidos por el dinero. Es precisamente en este campo del valor de cambio de la mercancía-guion donde ésta se muestra como un producto susceptible de autonomía y como una mercancía independiente de su traducción a la pantalla y de su conversión en contenido audiovisual. En primer lugar, tal y como hemos señalado con antelación, la mayoría de los escritores habitan una realidad profesional en la que obtienen su remuneración de la venta de guiones ${ }^{11}$. En segundo lugar, el cobro por un guion o por un proyecto de serie de ficción televisiva por parte de un escritor no implica que dichos materiales sean puestos en escena y se materialicen en una traducción audiovisual de los mismos. La mayor parte de los guiones que son fabricados por el trabajador colectivo de la escritura televisiva, así como la inmensa mayoría de proyectos de series de ficción confeccionados por guionistas, nunca llegan a la pantalla, ni siquiera son rodados. Unas veces las empresas productoras de televisión y las cadenas encargan y compran esos materiales, pero luego sólo seleccionan para su desarrollo y su fabricación una ínfima parte los mismos ${ }^{12}$. Otras veces son los propios guionistas los que operan como origen y motor del proyecto, a

\footnotetext{
${ }^{11}$ De hecho, suele ser común que el propio marco contractual que regula la actividad profesional de los escritores de ficción televisiva recoja explícitamente entre sus cláusulas el pago por guion finalizado y entregado.

${ }^{12}$ La producción cultural en su conjunto experimenta este tipo de fenómenos. Zallo (2007: 222) apunta que en el ámbito cultural la producción es mucho mayor que la oferta presentada al mercado: "o sea, hay una inmensa producción, una gran oferta múltiple invisible, una limitada oferta viva y una aún más limitada oferta visible y económicamente solvente".
} 
partir de un pitch inicial que se suele acompañar más adelante de la escritura de un argumento, los perfiles de los personajes, el guion del episodio piloto y el diseño del arco dramático y las continuidades de la primera temporada de la serie propuesta. En este segundo caso, no es inusual que los escritores ni siquiera vean remunerado su trabajo en la confección de los mencionados materiales.

En el marco de esta cartografía de las realidades de la escritura de contenidos de ficción televisiva serial, no sólo se pone de manifiesto el carácter independiente de la mercancía-guion, ajeno a todo carácter intermedio, sino que, lo que resulta todavía más importante, el propio valor de uso del guion aparece difuminado, pues la mayoría de las historias creadas por los escritores nunca llegan a una pantalla. Desde este punto de vista, la autonomía del guion descansa fundamentalmente en una particular materialización de la forma mercancía que tiende hacia la extinción del valor de uso de la misma.

En un contexto de este tipo, el grado enorme de capitalización de la escritura televisiva se mide, precisamente, por su carácter de escenario productivo en el que el valor de cambio es susceptible de independizarse por completo del valor de uso. Lo paradójico al respecto es que la naturaleza autónoma del guion se funda en la pérdida de utilidad del mismo, es decir que, dentro del marco actual de producción mercantil de contenidos televisivos, el trabajo concreto de escritura de series de ficción conquista su aparente autonomía mediante su reducción a trabajo abstracto ${ }^{13}$. Al evaporar el valor de uso del guion, la racionalidad productiva que gobierna la actividad profesional de los guionistas en el sector televisivo impone la consecución de independencia de su producto como pérdida del valor de uso de su trabajo. En este contexto, más allá de operar como vía de acceso al dinero, su trabajo para la mayoría de los escritores de contenidos seriales de ficción televisiva como carente de utilidad y de sentido.

\section{Conclusiones}

A lo largo del texto hemos propuesto una inmersión en el ecosistema de la fabricación de contenidos de ficción televisiva serial en el Estado español focalizando la atención en los escritores de dichos contenidos y en el producto de su trabajo: los guiones. En el curso de nuestra exploración hemos desvelado el carácter paradójico de ambos elementos, guionistas y guiones, tan imprescindibles para la confección de series televisivas como invisibles para los públicos mediáticos.

Nuestro viaje analítico en torno a la cualidad del guion ha partido de un anclaje semántico a partir del propio término y de los discursos implícitos en los modos de nombrar el producto del trabajo de los guionistas. Comenzando por la terminología generalizada en Hollywood, la primera industria cinematográfica del planeta, y

\footnotetext{
${ }^{13}$ Según Marx, la naturaleza dual de la mercancía, derivada de su carácter de contenedor de un valor de uso (utilidad) y un valor de cambio, se relaciona con una cualidad igualmente dual del trabajo productor de mercancías: trabajo concreto como tarea que produce el objeto en sí y su valor de uso, y trabajo abstracto en tanto que actividad productora del valor de cambio de la mercancía. "Todo trabajo es, de una parte, gasto de la fuerza humana de trabajo en el sentido fisiológico y, como tal, como trabajo humano igual o trabajo humano abstracto, forma el valor de la mercancía. Pero todo trabajo es, de otra parte, gasto de la fuerza humana de trabajo bajo una forma especial y encaminada a un fin y, como tal, como trabajo concreto y útil, produce los valores de uso" (Marx, 1981: 14).
} 
desembocando en la palabra guion en castellano, hemos observado explícitamente la connotación de una concepción de los guiones como productos intermedios carentes de sentido en sí mismos. El uso de las nociones scriptwriting o screenwriting en inglés y de la palabra guion en castellano, pone de manifiesto algo que Foucault señaló del lenguaje a partir de su análisis de la hermenéutica nietzscheana: que las palabras no indican un significado, sino que imponen una interpretación (Foucault, 1998: 276). En los propios modos de nombrar el resultado de la labor de los guionistas está implícita una concepción de dicho resultado que hace depender por entero su sentido de su traducción a la pantalla, dificultando de inicio toda concepción soberana del texto al que llamamos guion.

Más allá tanto de las visiones románticas de la escritura, como de las propuestas que han otorgado valor literario y carácter independiente a los guiones, nuestra categorización del guion de ficción televisiva serial como producto con sentido en sí mismo ha partido de un desplazamiento del análisis desde el interés por el guion como objeto, a la pregunta acerca del objeto del guion. En este sentido, nuestro razonamiento sobre la soberanía de los guiones televisivos propone sacar el ejercicio analítico del estricto terreno de la cualidad del objeto guion en sí mismo y su contenido, para rastrear la cualidad de las relaciones de producción en las que se inserta dicho objeto.

A partir de esta propuesta metodológica, dos han sido las ideas centrales que han resultado de nuestro razonamiento. En primer lugar, la relación entre la condición sistemáticamente infravalorada del guión en el ámbito de la ficción televisiva y la desvalorización de los guionistas en dicho ecosistema productivo, sujetos de una invisibilización notable y de una carencia de reconocimiento social que incide en el modo desfavorable en el que el colectivo profesional de escritores televisivos habita las relaciones de poder propias de las industrias mediáticas. En segundo lugar, a partir de las condiciones laborales concretas propias de la actividad del guionista en los circuitos de fabricación de series de televisión, en los que predomina el trabajo por cuenta propia, el guion aparece no sólo como producto de naturaleza plenamente mercantil, sino como una mercancía particular que en muchos casos, al no realizarse mediante su traducción a la pantalla, presenta un dudoso valor de uso. Es precisamente esta circunstancia la que nos ha llevado a proponer la paradoja de un carácter autónomo del guion que se funda en la no realización del mismo y en la pérdida de su sentido formal. 


\section{Referencias bibliográficas}

Balázs, B. (1970). Theory of the film: Character and Growth of a New Art. New York: Dover Publications.

Benjamin, W. (2004). El autor como productor. México: Itaca.

Bordwell, D., Staiger, J., y Thompson, K. (1985). The Classical Hollywood Cinema: Film Style and Mode of Production to 1960. New York: Columbia University Press.

Deleuze, G. y Guattari, F. (1994). Mil mesetas. Capitalismo y esquifrenia. Valencia: Pre-textos.

Dunne, P. (1980). Take Two: A Life in Movies and Politics. New York: McGraw-Hill.

Foucault, M. (1998). "Nietzsche, Freud, Marx". En M. Foucault, Aesthetics, Method, and Epistemology. Essential Works of Foucault, 1954-1984, Vol. 2 (pp. 269-278). New York: The New Press

Fundación Autor (2012). La escritura de guión en España. Un estudio de la profesión. Madrid: Fundación Autor.

Lara, A. L. (2013). Realidades de la ficción: bioproducción y trabajo cognitivo en la fábrica televisiva. La escritura de series de televisión en España: cualidades y condiciones de los trabajos y los trabajadores. Tesis doctoral defendida en la Universidad Complutense de Madrid en septiembre de 2013. Recuperado de http://eprints.ucm.es/23817/1/T34972.pdf

Lara, A. L. (2018). El narrador paradójico: invisibilidad y ambivalencia de la escritura de ficción televisiva en España. SERIES, International Journal of TV Serial Narratives, $I V(03)$.

Maras, S. (2009). Screenwriting. History, Theory and Practice. London: Wallflower Press.

Marx, K. (1981). El Capital. Crítica de la economía política. Tomo I. La Habana: Editorial de Ciencias Sociales.

Zallo, R. (2007). La economía de la cultura (y de la comunicación) como objeto de estudio. ZER Revista de Estudios de Comunicación, 12(22), 215-234. 